Rational Bureau of Standaros

Library, E-01 Admin. Bldg.

\title{
Experimental Techniques for Electron Scattering Investigations
}

U.S. 'ARTMENT OF OMMERCE National Bureau of Standards 
The National Bureau of Standards ' was established by an act of Congress March 3, 1901. Today, in addition to serving as the Nation's central measurement laboratory, the Bureau is a principal focal point in the Federal Government for assuring maximum application of the physical and engineering sciences to the advancement of technology in industry and commerce. To this end the Bureau conducts research and provides central national services in four broad program areas. These are: (1) basic measurements and standards, (2) materials measurements and standards, (3) technological measurements and standards, and (4) transfer of technology.

The Bureau comprises the Institute for Basic St andards, the Institute for Materials Research, the Institute for Applied Technology, the Center for Radiation Research, the Center for Computer Sciences and Technology, and the Office for Information Programs.

THE INSTITUTE FOR BASIC STANDARDS provides the central basis within the United States of a complete and consistent system of physical measurement; coordinates that system with measurement systems of other nations; and furnishes essential services leading to accurate and uniform physical measurements throughout the Nation's scientific community, industry, and commerce. The Institute consists of an Office of Measurement Scrvices and the following technical divisions:

Applied Mathematics-Electricity-Metrology-Mechanics-Heat-Atomic and Molecular Physics-Radio Physics -_Radio Engineering - Time and Frequency - Astrophysics "-Cryogenics."

THE INSTITUTE FOR MATERIALS RESEARCH conducts materials research leading to improved methods of measurement standards, and data on the properties of well-characterized materials needed by industry, commerce, educational institutions, and Government; develops, produces, and distributes standard reference materials; relates the physical and chemical properties of materials to their behavior and their interaction with their environments; and provides advisory and research services to other Government agencies. The Institute consists of an Office of Standard Reference Materials and the following divisions:

Analytical Chemistry-Polymers-Metallurgy_Inorganic Materials_Physical Chemistry.

THE INSTITUTE FOR APPLIED TECHNOLOGY provides technical services to promote the use of available technology and to facilitate technological innovation in industry and Government; cooperates with public and private organizations in the development of tcchnological standards, and test methodologies; and provides advisory and research services for Federal, state, and local government agencies. The Institute consists of the following technical divisions and offices:

Engineering Standards - Weights and Measures — Invention and Innovation — Vehicle Systems Research_Product Evaluation-Building Research_Instrument Shops-Measurement Engineering-Electronic Technology-Technical Analysis.

THE CENTER FOR RADIATION RESEARCH engages in research, measurement, and application of radiation to the solution of Bureau mission problems and the problems of other agencies and institutions. The Center consists of the following divisions:

Reactor Radiation-Linac Radiation-Nuclear Radiation-Applied Radiation.

THE CENTER FOR COMPUTER SCIENCES AND TECHNOLOGY conducts research and provides technical services designed to aid Government agencies in the selection, acquisition, and effective use of automatic data proccssing equipment; and serves as the principal focus for the development of Federal standards for automatic data processing equipment, techniques, and computer languages. The Center consists of the following offices and divisions:

Information Processing Standards-Computer Information - Computer Services - Sys-

tems Development-Information Processing Technology.

THE OFFICE FOR INFORMATION PROGRAMS promotes optimum dissemination and accessibility of scicntific information generated within NBS and other agencies of the Federal Government; promotes the devclopment of the National Standard Reference Data System and a system of information analysis centers dealing with the broader aspects of the National Measurement Systcm, and provides appropriate services to ensure that the NBS staff has optimum acccssibility to the scientific information of the world. The Office consists of the following organizational units:

Officc of Standard Refcrencc Data-Clcaringhouse for Federal Scientific and Technical Information -Office of Technical Information and Publications-Library-Office of Public Information-Officc of International Relations.

Hadquarters and Laboratories at Giathersburk. Maryland, unless otherwise noted: mailing address Washington. D.C. 20234 


\title{
UNITED STATES DEPARTMENT OF COMMERCE
}

Maurice H. Stans, Secretary

NATIONAL BUREAU OF STANDARDS - Lewis M. Branscomb, Director

\section{NBS TECHNICAL NOTE 523 \\ ISSUED APRIL 1970}

Nat. Bur. Stand. (U.S.), Tech. Note 523, 46 pages (Apr. 1970) CODEN: NQBTNA

\section{Experimental Techniques for Electron Scattering Investigations}

\author{
Samuel Penner \\ Linac Radiation Division \\ Center for Radiation Research \\ National Bureau of Standards \\ Washington, D.C. 20234
}

\begin{abstract}
NBS Technical Notes are designed to supplement the Bureau's regular publications program. They provide a means for making available scientific data that are of transient or limited interest. Technical Notes may be listed or referred to in the open literature.
\end{abstract}

For sale by the Superintendent of Documents, U.S. Government Printing Office, Washington, D.C., 20402. (Order by SD Catalog No. C 13.46:523). Price 50 cents. 



\section{FOREWORD}

This report was originally prepared for presentation at a Seminar on the subject "Nuclear Investigations on Linear Electron Accelerators at Low and Medium Energy"' held at the P. N. Lebedev Physical Institute, Moscow, USSR, December 10-16, 1969. The paper presents a fairly extensive review of modern techniques used in the performance of electronnucleus scattering experiments with electron 1 inear accelerators in the 50 to $1000 \mathrm{MeV}$ range, and particularly of the NBS facility which has not been fully described elsewhere. The paper is therefore reproduced here in its original form in order to be available to a wider audience. 
CONTENTS

Page

1. Introduction.....................................

2. Spectrometer Design..................................

3. Detector Systems ...................................

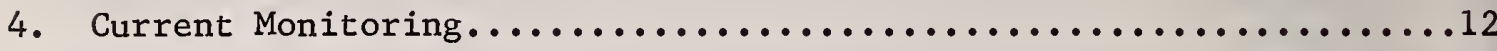

5. Background Considerations............................... 14

6. Data Handling with On-Line Computer Systems................16

7. Theoretical Considerations.............................19

8. Electron Scattering at $180^{\circ} \ldots \ldots \ldots \ldots \ldots \ldots \ldots \ldots \ldots \ldots \ldots \ldots \ldots \ldots \ldots$

9. Summary.......................................... 24

10. References....................................... 26 
Experimental Techniques for Electron Scattering Investigations

\section{S. Penner}

Modern experimental techniques for high energy electron scattering are discussed. Subjects included are: high resolution spectrometer design, the energy loss spectrometer concept, detector ladder systems, beam current monitoring techniques, suppression and measurement of background, the use of on-1ine computer systems, theoretical considerations and the analysis of data, and $180^{\circ}$ scattering.

Key words:background; current monitoring; detector ladders; electron scattering; experimental techniques; 1ine-shape fitting; on-1ine computer systems; radiative tails; resolution; spectrometer design.

\section{Introduction}

For many years it has been said that electron scattering is a powerful tool for the study of nuclear structure, because quantum electrodynamics is a very accurate theory and because the weakness of the electromagnetic interaction allows accurate theoretical interpretation of experimental results. In spite of these advantages, electron scattering has in the past contributed little to our understanding of the nucleus, in contrast to the vast amount of information obtained by reaction studies employing nuclear particles (protons, alphas, etc.). This situation has changed greatly in recent times, and electron scattering is at last proving its great value as an experimental technique for the study of nuclear properties.

As a result of recent improvements in experimental techniques and apparatus we are now able to perform detailed and accurate experiments which yield valuable information on nuclear structure. For example, figure 1 shows a recently-obtained spectrum of electrons inelastically scattered from $\mathrm{Ti}^{48^{\underline{1} / *}}$ compared to an inelastic alpha scattering experi*Literature references at the end of this paper. 
ment on the same nucleus. We can see from figure 1 the the resolution, peak-to-background ratios, and the states excited in the two experiments are quite comparable.

The main reasons that we are now able to perform experiments of this quality are: (1) the development of the modern electron 1inear accelerator which provides high current, small beam energy spread, good beam emittance, and improved duty cycle compared to earlier machines; better understanding of the principles of beam transport which allows us to obtain wel1 resolved, stable electron beams of high quality ${ }^{2 /}$; improvements in the design and construction of magnetic spectrometers to obtain high resolution and good acceptance (solid angle times useful momentum range along the focal plane); (4) the development of multichannel "ladder" detector systems; (5) improved methods of beam current monitoring; and (6) the use of on-1ine computer systems, enabling us to handle the enormous quantity of data obtained and to effectively control and monitor the progress of the experiment.

In this paper we will report on the present state-of-the-art in the experimental techniques mentioned above. We will also refer briefly to developments in electron scattering theory as they apply to the interpretation of experimental data, and discuss some current problems such as the treatment of backgrounds.

\section{Spectrometer Design}

The properties of an electron scattering facility are largely determined by the parameters of the magnetic spectrometer which is used. We will begin our discussion by 1 isting the major design requirements for a spectrometer. First, good resolution is essential. As shown in Table I, 
many factors contribute to the overall resolution of the system. Not only must the intrinsic resolution of the spectrometer be good, but large dispersion is required so that detectors and beam spots of reasonable dimensions can be used. Second, because low cross sections are an ever-present problem, large solid angle and momentum acceptance are needed. Third, the spectrometer should be useable over a broad range of both momentum and scattering angle to permit measurement of both longitudinal and transverse form factors over an extended range of momentum transfer. Fourth, the geometry of the spectrometer must allow for good shielding of the detector system because we must measure very small cross-sections in the presence of very high backgrounds.

Ten years ago the standard instrument for electron scattering was the $180^{\circ} \mathrm{n}=1 / 2$ double focussing spectrometer ${ }^{3 /}$. It provided a resolution of 0.1 to $0.2 \%$ with a solid angle of order .005 steradians and, since the double-focussing property results in a small detector volume, it was relatively easily shielded. With the advent of the high current electron linac, we could afford to define a smaller momentum bin in the beam transport system, use thinner targets to reduce energy-loss straggling, and still have reasonable counting rates. It was then necessary to design spectrometers to take advantage of the better resolution capability thus available. We chose the "magic angle spectrometer" provides good resolution while retaining all the desirable properties of the $180^{\circ}$ spectrometer. This spectrometer, shown in figure 2 , is now in use at NBS. Similar spectrometers are in use at Amsterdam ${ }^{5}$, Tohoku (Japan), and Glasgow. A spectrometer of slightly modified design providing smaller aberrations in the transverse (scattering) plane and a larger 
useful momentum acceptance is being installed at Saclay ${ }^{6}$.

The resolution obtainable with the magic angle spectrometer is demonstrated in figure 3, which shows inelastic scattering data from the first two excited states of $0^{16}$ from a $35 \mathrm{mg} / \mathrm{cm}^{2}$ beryllium oxide target $\underline{\text { ? }}$. The overall resolution of about $60 \mathrm{keV}$ (full width at half-maximum) results from a total instrumental resolution of $48 \mathrm{keV}(0.08$ percent of the incident energy) plus a contribution due to energy loss straggling. I do not want to give the impression that the magic angle spectrometer is the only design which is suitable for high resolution electron scattering work, because there are in fact many other possibilities. I want to mention here one entirely different design, the "energy loss spectrometer" being built at MIT 8 ! The MIT spectrometer is a uniformfield, $90^{\circ}$ deflection split-pole type, designed for a momentum resolution capability of about one part in $10^{4}$ over a $2 \%$ interval, and a solid angle of about $5 \mathrm{~ms} \mathrm{r}^{9}$. The important difference of the MIT design from conventional spectrometers is that the transverse optics provides "point-toparallel" rather than "point-to-point" focussing. The reason for this choice is that for large momentum transfers and 1 ight target nuclei the kinematic broadening of the resolution for a reasonable acceptance angle in the scattering plane is large. By employing point-to-parallel focussing, knowledge of the scattering angle is preserved, and thus a correction can be made for the kinematic broadening. A possible disadvantage of this approach is that one of the methods for handling the "instrumental background" is not possible here. This will be further discussed in section 5 .

While we are discussing the MIT design, I would like to comment on 
the energy-loss system which the MIT group plans to use. The basic principle is illustrated in figure 4, which is taken from reference 8 . In this system, the beam transport arrangement provides a momentumdispersed (but spacially focussed) beam at the spectrometer target location. If this dispersion is properly matched to the imaging and dispersion properties of the spectrometer then all electrons which have lost a given amount of energy in the target arrive at a single point in the spectrometer focal plane, independently of their initial energy. Since good resolution in energy loss is the essential requirement in electron scattering, the energy loss system allows one to use a larger portion of the current from the linac while maintaining good resolution. Thus the advantage of this system is that it permits much higher counting rates whenever beam current is the limiting factor. I want to emphasize that the energy loss system does not allow a relaxation in the resolution requirements of either the beam transport system or the spectrometer. In fact, it may be more difficult to obtain the needed resolution because some spectrometer aberrations, which are not significant when the beam spot size at the target is small, are important for the larger spot size of the dispersed beam.

There are some general principles of spectrometer design which are important regardless of the specific optics. In order to obtain the optical properties called for in the design it is necessary to obtain the required magnetic field configuration to a high degree of accuracy 10 /. Obtaining the desired field configuration is particularly difficult in electron scattering because the spectrometer must be useable for a large range of particle momenta, and thus a large range of field strengths. 
Differential saturation of the iron can distort the field distribution drastically when the field strength is changed. One method of minimizing this effect is to use the "Rogowski profile" for the edge of the magnet poles 11/. To simp1ify the construction problem, it may be adequate to approximate the Rogowski profile by one or two plane segments at the edges. This is the procedure used in the NBS spectrometer, as shown in figure 5. We have shown that the optical properties of this spectrometer do not change appreciably in the range of field strengths between $0.9 \mathrm{kG}$ and $13 \mathrm{kG}$ (in our case this corresponds to momenta between about 20 and $290 \mathrm{MeV} / \mathrm{c}$ )

In order to fully use the high resolution properties of the spectrometer, the magnetic field must be stable to a small fraction of the resolution width. Stabilities of one part in $10^{5}$ or better are obtained from conventional well regulated power supplies. Figure 6 demonstrates the current stability of the NBS spectrometer. When taking data we routinely measure the spectrometer current (actually the voltage across a series "shunt" resistor held at constant temperature by water cooling) along with our count rate measurements at intervals of typically one to three minutes. We do not routinely measure the magnetic field in the spectrometer directly as is done in most other laboratories. As a matter of fact it is not obvious that a single point magnetic field measurement should be more precisely proportional to electron momentum than is a current measurement. Our procedure is to follow a prescribed path along the hystersis curve of the iron by "cycling" the spectrometer. This provides a reproducibility of momentum for a given spectrometer current within one or two parts in $10^{4}$ when the spectrometer is recycled 12 ! When taking 
typical inelastic scattering spectra, peaks corresponding to known energy levels are observed to occur at the proper electron energy, typically within $\pm 5 \mathrm{keV}$.

\section{Detector Systems}

The main requirements placed on detector systems for scattered electrons are: small size in the dispersion direction to take full advantage of the resolution, stability of counting efficiency, good rejection of background events, and the ability to operate at high instantaneous counting rates. It is highly desirable to use many counting channels, not only to improve the data accumulation rate, but to minize crosssection errors due to variations of beam energy, spectrometer magnetic field, or beam current. The first multichannel detection system, or "1adder counter" for electron scattering work was developed at Stanford by H. W. Kenda11 13 ! The basic idea is that a number of small detectors are located along the focal plane of the spectrometer in order to detect the position at which each electron intersects the focal plane. Thus each detector corresponds to a slightly different momentum bin of scattered electrons for any given magnetic field in the spectrometer.

The ability to handle high counting rates (in each detector) is very important in electron scattering for two reasons. First, it is often necessary to obtain good statistical precision for the net area of a smal1 peak superimposed on a large continuum (e.g., due to the radiative tail of the elastic peak). Secondly, it is often desirable to normalize inelastic scattering cross sections to the elastic scattering cross section which may be many orders of magnitude larger. Better accuracy can be obtained when it is possible to avoid making large changes in the 
beam current between the elastic and inelastic measurements.

Several different types of detectors have been used successfully in ladder systems, including plastic scintillators, semiconductors, and multi-wire spark chambers or proportional counters. We will describe these types of ladder counters briefly here and compare the advantages and disadvantages of each type. We refer to the literature for more detailed descriptions.

Plastic scintillator ladders offer the advantages of high count rate capability due to the fast response and rapid recovery of the scintillator-phototube combination. When a coincidence requirement between the counters in the ladder and a "back-up" detector (usually a large Cerenkov Counter) is included in the system, the background rejection properties are good. However, it has been demonstrated that if sufficient care is taken in reducing the background, plastic scintillator detectors can be used effectively with no coincidence requirement $\frac{14 / \text { (at }}{}$ least when the electron beam energy is low). The main problem with plastic scintillators in high resolution systems is that the detectors must be very small, making it difficult to get enough light to the phototube to have a satisfactory signal-to-noise ratio. It is also difficult to package a scintillator system compactly, because of the necessity for light pipes and the relatively large size of phototubes which should be as close to the scintillators as possible.

Counter ladders employing lithium-drifted silicon semiconductor

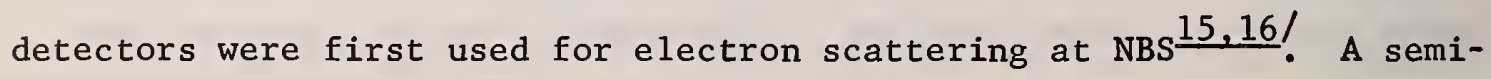
conductor ladder is also in use at the Tohoku linac, and a large semiconductor system is in preparation at Saclay. It is relatively easy to 
make semiconductors very small (the dimensions of the present NBS detectors are $1 \times 1.25 \times 60 \mathrm{~mm}^{3}$ ), and pack them close together in a ladder. Since appropriate preamplifiers can be made very small, expecially if integrated circuits are used, the entire array is quite compact compared to a scintillator-phototube system and therefore is easier to shield. Semiconductor systems are in general not as fast as phototube systems, but if the detectors are cooled to liquid-nitrogen temperature, charge collection times of about 20 ns can be obtained. The NBS counters have a coincidence resolving time of $50 \mathrm{~ns}$ and a dead time of $80 \mathrm{~ns}$. With the aid of auxiliary circuitry to measure chance counts and dead-time corrections, our system is useable at high counting rates and in severe background-to-true count rate situations 12,15 ! Two disadvantages of semiconductor detectors are their sensitivity to radiation damage and to surface contamination. Because of these effects, the detector efficiencies can change (over a period of some weeks or months), and eventually the detectors must be replaced. This is not too serious because the detectors themselves are inexpensive, and replacing them is not difficult. In the NBS system, the useful detector lifetime seems to be roughly one to two years.

Spark chambers of various types have also been employed as ladders counter 17,18 ! The spatial resolution of spark chambers is about the same as semiconductor detectors; they are jus t as compact, and the electronic readout system is simpler. A great many channels can be built since the cost per channel is relatively small. Counting rate limitations and poor background rejection seem to be the main disadvantages of spark chambers. I am not aware of any system of this type which is capable of 
recording more than one count per linac beam pulse per channel, whereas phototube systems and even semiconductors can record several events per pulse for beam pulse lengths of a few microseconds $\underline{39 /}$.

A serious problem with any multichannel array is to determine the detection efficiencies of the individual detectors. The problem is severe with detectors of very small size because a substantial fraction of the incident particles pass through or near the edges, thus producing pulses which are smaller (and perhaps of a different shape) than the pulses due to particles passing through the center of the detector. Unless one is willing to be committed to a data taking procedure in which a11 detectors in the ladder are eventually located at all electron momemtum values in the spectrum desired, a means must be found for accurately intercomparing the efficiencies of all detectors. The usual procedure for obtaining the efficiencies is to measure the counting rates due to a flat, or at least reasonably smooth, spectrum of scattered electrons. From data taken in all detectors at several closely spaced spectrometer field settings, the relative efficiencies of the detectors can be determined 19 ! A different procedure can be used when provision is made for mechanically moving the detectors along the focal plane. One simply moves the ladder so that each detector occupies the position previously occupied by its neighbor and compares the counting rates of each pair of

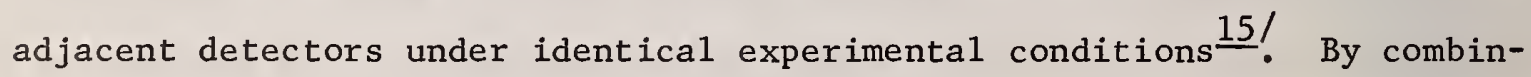
ing these two procedures it is possible to distinguish intrinsic differences between the detectors from a number of contributing effects such as variation of solid angle and dispersion along the focal plane and loss of efficiency due to multiple scattering of electrons in the small detectors 
(resulting in some electrons not reaching the back-up detector, an effect which can be important for low energy electrons).

The ability to move the detectors along the focal plane has other useful features. When the resolution is good, only a few detectors see a peak in the spectrum at one magnetic field setting. For an isolated leve1, this is not very important since only the area of a peak is physically significant, but for closely spaced levels or small peaks superimposed on a continuum, many points closely spaced in energy are needed to distinguish contributions from each level and/or the continuum. With a movable ladder, it is easy to space data points very closely by sma11 changes in the detector position. Of course, such changes can also be accomplished by changing the magnetic field but is seems to us to be easier to mechanically move the detectors a precisely determined distance reliably and reproducibly. In the NBS system, the precision of detector motion corresponds to 2 parts in $10^{6}$ in momentum, which would be very difficult to achieve or measure with magnetic field changes.

A separate, but related, problem is the determination of the absolute efficiency of the detector system which is necessary if absolute cross sections are to be measured. Absolute electron scattering cross sections are rarely measured, but in fact can be traced back to the assumed accuracy of the Rosenbluth formula for elastic electron-proton scattering together with the experimentally known proton form factors $\frac{20 /}{\text {. }}$ At NBS we have demonstrated the feasibility of a method for determining the absolute efficiency of our detector system which involves the use of an auxiliary "area" detector in the focal plane which is much larger in the dispersion direction than the detectors in the ladder. The area detector 
determines the area of a scattering peak without precise knowledge of detector edge effects, since only a very small fraction of electrons in the peek pass near the edges. The small detectors can be compared to the area detector by a series of measurements in which the detectors are moved along the focal plane. From this data the efficiencies of the sma11 detectors are determined relative to the area detector, and the small influence of edge effects on the latter can be removed iteratively. To complete the determination of absolute efficiencies, it is necessary to study in detail the response of the area detector as well as the plastic scintillators in coincidence with it. This is done by determining pulse height spectra, variations of count rates with detector collection voltage or phototube high voltage, and by other standard techniques.

\section{Current Monitoring}

Accurate beam charge measurement is essential to any cross section determination. A Faraday cup together with an electronic integrating circuit provides good absolute accuracy, but introduces several problems if used during the course of the scattering measurements. The problems are: (1) the cup is a very strong source of background; (2) at low energies, multiple scattering in the target may prevent some of the electrons in the beam from reaching the cup; and (3) if used continuously, the cup must be cooled to remove the heat due to the beam power. Because of these difficulties, most laboratories employ other types of current monitors, which must be calibrated against the Faraday cup. The two most commonly used monitors are the secondary emission monitor (SEM), and the ferrite pulse transformer. Although good results have been reported with the SEM 21 /, we have not had much success with them at NBS. 
We believe that our high beam current causes changes in the surface properties of the foils in the SEM, resulting in erratic behavior and substantial variations in calibration during data taking.

A ferrite-core pulse transformer provides a good measurement of beam pulse current, and produces no background since the beam is not intercepted. Electronic integration of the signal to obtain the beam charge is more difficult than in the case of a Faraday cup because the signals are smaller and A. C. coupled. Appropriate integrating circuits have been developed $\underline{22 /}$ which provide charge measurement precision of about \pm 0.2 percent for currents of the order of $0.5 \mu \mathrm{A}$ or larger. The limiting factor seems to be how well electrical pickup (primarily due to the linac modulators) can be suppressed $\frac{23}{\text { ! }}$.

An interesting technique for current measurement is in use at Amsterdam, employing a ferrite pulse transformer together with a Faraday cup 24 . The Faraday cup is located far enough from the experimental target so that it can be well shielded. A pair of quadrupole magnets collects the multiple-scattered electron beam from the target to maximize the fraction of the beam reaching the cup. A known multiple of the current collected by the cup is passed back through the ferrite so as to cancel the beam current pulse $\mathrm{25}^{25}$. A measurement of the charge collected by the Faraday cup together with a knowledge of the multiplying factor which produces a null signal from the ferrite provides an accurate measure of the beam charge delivered to the target. This method is illustrated in figure 7.

A similar procedure is used at NBS to measure the absolute charge collection efficiency of our Faraday cup. Measurements now being made 
by J. S. Pruitt of our laboratory indicate that the cup, whose dimensions

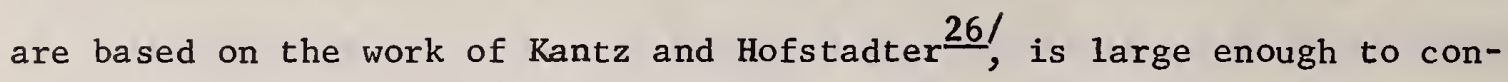
tain essentially all of the charged secondaries produced by the beam. He also finds that ther is a small $(<1 \%)$ but energy-dependent loss of charge due to backscattering of electrons through the entrance of the cup, and that there is a small ( . .5\%) increase in charge collected in the cup due to collection of secondary electrons generated by electron-electron scattering in the entrance vacuum window of the cup. By applying corrections for these measured effects, we believe we can determine the absolute efficiency of our Faraday cup to an accuracy of better than $0.1 \%$.

\section{Background Considerations}

Suppression of background counts in the detector system is essential for accurate cross-section measurements. We distinguish three main sources of background which must be considered.

First, there is the room background generated primarily at the place the electron beam, having passed through the target, is finally absorbed. This type of background is handled by shielding the Faraday cup (as mentioned already in section 4), or by using a non-intercepting monitor (or SEM) and carrying the residual beam to a well shielded "beam dump". Shielding directly around the detectors is obviously necessary also. At NBS our main shielding consists of $20 \mathrm{~cm}$ of 1 ead and $10 \mathrm{~cm}$ of boron loaded polyethylene. The room background which does penetrate the shielding is mostly neutrons and thus produces many more singles counts in the individual detectors than it does coincidence counts which might be interpreted as good events. Thus the main effect of room background in our case is to produce electronic dead times. This amounts to roughly $2 \%$ 
dead time per microamp of beam current when the Faraday cup is in the beam, and $0.5 \%$ per microamp when the cup is not in the beam. We feel that we can make deadtime corrections to better than $20 \%$ of their value, thus permitting $1 \%$ counting accuracy at $10 \mu \mathrm{A}$ beam current.

A second source of background is degraded electrons and $x$-rays in and around the main electron beam, which can scatter from the target and enter the spectrometer, besides contributing to the room background 27 ! Suppression of this type of background depends mainly on good beam handling technique 2 .

The third and most serious type of background is that due to scattered electrons which enter the spectrometer but have an energy such that they can not reach the detectors directly, but instead scatter from the pole tips and vacuum chamber walls of the spectrometer. This "instrumental scattering" background is worst when attempting to measure a smal1 inelastic peak which is a few percent (in scattered electron energy) away from a strong elastic scattering peak. This type of background can be reduced by at least three approaches as follows: (1) A system of baffles is installed in the spectrometer to reduce the number of electrons striking the vacuum chamber walls and to absorb the scattered electrons which

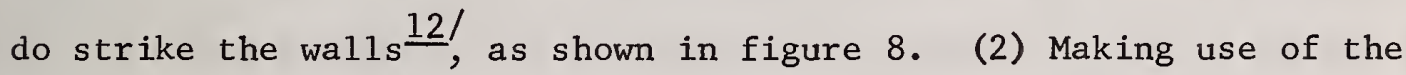
double-focussing property of the spectrometer one moves the detectors transversely (out of the spectrometer midplane) so that electrons coming from the target do not pass through them, and measures the background directly 14 ! Obviously this method is not available if the spectrometer optics does not produce a transverse-plane image of the target in the focal plane, as in the MIT system discussed above (in section 2). (3) The 
instrumental scattering is greatly reduced by using a thin-walled vacuum chamber and moving the magnet return yoke away from the immediate vicin-

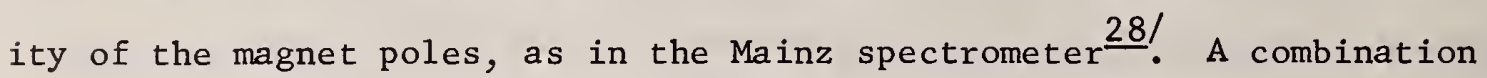
of these methods should be better than any one of them. Therefore at NBS, where we use the baffle technique, we plan to replace our present detector ladder with a two dimensional array so that we can simultaneously measure good events and instrumental background.

6. Data Handling with On-Line Computer Systems

In high resolution electron scattering, enormous quantities of data must be recorded and analyzed. In a typical experiment on the low-lying levels of a light nucleus, one would spend perhaps six hours to obtain a spectrum for a single incident energy. In order to obtain data at sufficiently closely spaced momentum intervals and to obtain data for several detectors at each momentum value (to be able to extract relative detector efficiencies as discussed in section 3 , and to make the results relatively insensitive to the accuracy of the efficiency intercomparison) we would use some 200-300 different combinations of magnetic field setting and detector ladder position. A typical spectrum of this type is shown in figure 9. At NBS we have a 20 channel ladder, so that for each of these 200 or so "runs" we must record 40 numbers (including such quantitites as beam charge, run time, spectrometer shunt voltage, counts in auxiliary channels for dead time corrections, etc., in addition to the counts in 20 channels). I can say from experience that it would just not be feasible to take data of this type without some type of automatic data hand 1 ing equipment.

In addition to collecting the data, the computer serves several 
other important functions. It can be programmed to perform a set of runs automatica11y. For example, the computer at NBS monitors the beam charge continuously and terminates the run (by turning off a master counting gate) when a predetermined charge has been collected. It then computes the results of the run, types out the data and records them on magnetic tape for further use, clears all temporary data storage, moves the detectors and/or changes the spectrometer field as desired, and turns on the master counting gate for the next run. The experimenter, being freed of al1 these routine operations, has time to study the results as they are obtained and thus can intelligently determine the best way to proceed with the experiment. In order to make these decisions, the experimenter should have the results available on-line. This means that the computer must analyze the data during and between runs. At NBS we can choose any data field in the computer for observation as an oscilloscope display. We might choose to look at the counts in the detectors of the ladder displayed as a histogram while the data are being accumulated. More often, we would watch one of several spectra, corresponding to different targets or to analysis of one spectrum in various size momentum bins. These spectra are updated by the computer after each run. The data of each run are corrected for such things as counting rate effects and detector efficiency differences and added to the previously-existing spectra in units of counts per MeV per unit beam charge. A typical spectrum of this type is shown in figure 10. These are actually the same data as in figure 9 except that the results from all detectors has been combined and divided into uniform momentum bins. This particular spectrum was obtained by off-1ine reanalysis of data on a different computer, 
using as input a punched-paper tape record produced by the on-1ine system. Reanalysis is necessary because detector efficiencies and backgrounds are determined during the course of the experiment, so that the on-line analysis relies on provisional values based on past experience which may not be highly accurate. Several different computers have been used for reanalysis, including the on-1ine system.

I do not want to describe any particular computer system in detail, however I would like to mention some of the general features of the NBS system 29 . We chose a modular approach to the programming, as we11 as to the hardware, in order to have a system with maximum flexibility. The system is used for al1 the physics experiments done with our linac including neutron time-of-flight spectroscopy, charged particle electroproduction experiments (such as $(\gamma, \mathrm{p})$ and $(\gamma, \mathrm{d}))$, and several others besides electron scattering. Very little programming must be done for any particular experimental setup because with the modular concept most programs (executive system, display of various kinds, etc.) are used by 211 experiments. Most changes of experimental procedure or equipment do not require additional programming. For example, when we converted our detector ladder from 12 to 20 channels, no original programming was needed, although several simple modifications in existing programs were made to accomodate the additional channels of information. The multilevel priority interrupt feature of our system is extremely valuable. It permits several experimenters to use the computer simultaneously. (Although possible, this is not actually done very much because of memory size limitations, and only one experiment is usually done with the linac beam at one time.) More important, the interrupt feature permits assign- 
ment of priorities to the many functions performed by the computer in one experiment. For example, a11 data taking functions are assigned higher priority than the display function, and the magnetic tape data recording is done at higher priority than the typing of results since the tape record is more useful than the printout.

Fina11y, I should like to point out that on-1ine computers for experiments with pulsed accelerators need not necessarily be extremely fast because the time between beam pulses is available for computation. The computer at NBS has a memory cycle time of $8 \mathrm{us}$ (the memory is 16,000 - 24 bit words). In electron scattering, about one millisecond is used between beam pulses for data accumulation, and about 5 seconds between runs for calculation of spectra, recording of data on tape, and changing of experimental conditions.

\section{Theoretical Considerations}

Although the subject of this paper is experimental techniques, there are certain theoretical questions which bear directly on the analysis and interpretation of experimental data, and therefore should be mentioned. These questions are more urgent now than they would have been a few years ago because of the increasing accuracy of recent experiments.

Radiative corrections are discussed in detail in the recent review

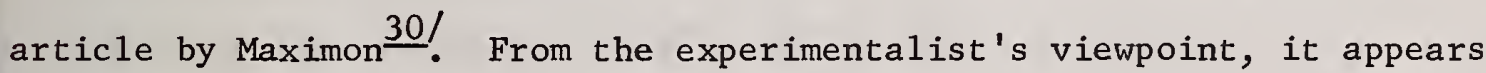
that the corrections are we11 enough understood for the reliable interpretation of experimental data at the level of about one percent accuracy. To go beyond this level of accuracy seems to be very difficult theoretically, and also difficult to verify experimentally. The reason it is difficult to verify the corrections experimentally is that even with the 
best resolution presently available, the largest contribution to the correction occurs at values of the radiative energy loss within the experimental resolution width, and thus is not directly accessible experimentally. It will be interesting to test the validity of the corrections with the improved resolution expected at the new Saclay and MIT facilities. (The only experimental test $I$ know of is to measure the area of a scattering peak as a function of the integration end point $\Delta E$, calculate the radiative correction for each value of $\Delta E$, and check for consistency) Dispersive corrections can be important in both elastic and inelastic scattering, particularly in the vicinity of diffraction minima $30 /$. I know of no unambiguous experimental measurements of dispersive effects, although there is one experiment in progress which might turn out to show an effect. Accurate elastic scattering measurements on the titanium

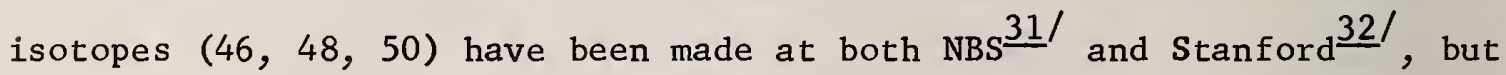
the momentum transfer corresponding to the first diffraction minumum was obtained by quite different combinations of scattering angle and beam energy in the two experiments. Dispersive effects are expected to be energy dependent and might be expected to be particularly large in this case because of the presence of very strong collective E2 transitions to low-lying excited states in these isotopes. The inelastic cross section reaches a peak at momentum transfer values near the first elastic cross section minimum.

As we continue to improve experimental resolution capability, we will attempt to measure the cross-sections for more closely spaced nuclear levels. Thus we will continue to be faced with the problem of determining the separate cross-sections of peaks in the spectrum which 
are not fully resolved. To do this accurately requires a line-shape analysis. I want to briefly describe the procedure developed by J. C. Bergstrom 7,33/. The calculation includes the effects of Landau straggling (ionization energy losses), small angle bremsstrahlung, radiation emitted during the large angle scattering, and the experimental resolution. The computer program which has been developed finds the best fit to one or more peaks in the spectrum plus continum (radiative tails and background). The parameters which can be found by the least squares analysis are peak height, peak energy location, target thickness (in terms of Landau straggling width), and resolution width (assumed to be a gaussian function). Alternatively any one or more of these parameters may be fixed, allowing the program to find best values for the remaining ones. For example, if the difference in energy of two levels is accurately known, the peak heights can be determined more accurately. In the case of levels which have a physical width comparable to or larger than the resolution, the physical width can be determined separately if the experimental resolution is known (say from the analysis of a nearby narrow peak in the spectrum). The curves in figures 3 and 11 are generated by this line-shape program.

In order to determine inelastic scattering cross-sections the radiative tails due to all processes at lower excitation must be subtracted from the observed spectrum. This subtraction is relatively unimportant in the case of resolved peaks, but must be done very we11 to obtain accurate cross section information in the continuum region (for examples, the giant dipole resonance and quasi-elastic scattering). The best available radiative tail calculations ${ }^{34 /}$ are performed in Born approxi- 
mation. These calculations give excellent agreement with experimental results for the first few $\mathrm{MeV}$ of the spectrum in the case of carbon targets with incident energies of 50 to $150 \mathrm{MeV}$, and scattering angles as large as about 160 degrees $14,27 /$. When thick target effects (bremsstrahlung and electron-electron scattering) are included, the agreement between calculation and experiment is within about $3 \%$ in the above-mentioned limited range of parameters. More important is the question of whether the calculated tail is correct for the entire spectrum. Figure 12 illustrates a case where the agreement appears to be better than $10 \%$ for the entire spectrum. In this example the absolute scale of the calculated tail is based solely on the observed elastic scattering cross section; there is no arbitrary normalization. This spectrum was measured with an incident energy of $69 \mathrm{MeV}$ from a carbon target at a scattering angle of $75^{\circ}$. At higher energies and more

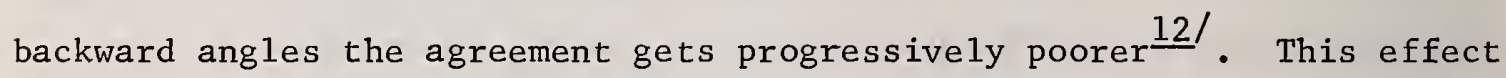
is not necessarily due to a failure of the radiative tail theory but could just as well be caused by the increasing importance of backgrounds and the relatively greater importance of the nuclear continuum cross section. Very little is known about the validity of radiative tail calculations in heavier nuclei, where the Born approximation may not be very good. More work in this area, both theoretical and experimental, is needed in order to understand the situation.

Until rather recently, electron scattering cross sections of 1 ight nuclei were conventionally analyzed in the framework of the Born approximation using plane-wave electron wave functions. (The failure of the Born approximation per se is discussed above where the effect is refer- 
red to as dispersive corrections.) Even in the lightest nuclei however the plane wave approximation is only good to about ten percent. Futhermore the distorted wave corrections depend on the multipolarity of the transition and thus do not cancel in the ratio of inelastic to elastic cross sections. A number of investigators have studied the problem of distorted wave effects $\frac{35}{}$, and accurate computer programs have been developed to perform the calculations by phase-shift analysis using relativistic coulomb wave functions, for both elastic and inelastic cross sections. In comparing experimental results, one must be sure to understand how each experiment was analyzed. We should be very careful when presenting experimental data to state very explicitly when and how distorted wave corrections are made.

\section{Electron Scattering at $180^{\circ}$}

In my opinion there has been a tendency among some of our colleagues to overestimate the value of $180^{\circ}$ scattering experiments. The reason for this is basically that the cross sections due to transverse effects (magnetic or transverse electric) do not increase at backward angles (at fixed momentum transfer the cross section actually decreases by about $30 \%$ between $150^{\circ}$ and $180^{\circ}$ ); thus when the scattering angle is large enough that errors due to subtracting continuum tails from the transverse cross section peaks are unimportant, there is no point in going to more backward angles. The optimum angle depends on resolution, counting rate, and background as well as on the ratio of transverse to coulomb cross sections (which we know to be essentially $\tan ^{2} \theta / 2$ ). My point is that we can do better in terms of resolution, counting rate, and background at conventional angles. The reasons are: (1) Up to about $150^{\circ}$ 
targets can be oriented for transmission geometry rather than the reflection geometry needed for $180^{\circ}$ scattering, giving for a fixed target thickness, a factor of nearly four in the effective thickness (hence, counting rate), and simultaneously better energy resolution because in transmission geometry only the Landau straggling contibutes to the energy spread, instead of the spread in energy loss corresponding to twice the target thickness which occurs in reflection. (2) It is usually necessary to severely limit the solid angle in $180^{\circ}$ experiments.

Scattering experiments at $180^{\circ}$ are valuable as a part of a more general program, for those special cases where transverse and large coulomb cross sections are present at the same excitation energy. I refer here particularly to magnetic elastic scattering $\stackrel{36 /}{\text {, }}$, and certain special cases of inelastic scattering such as the M1 contribution to the electrodisintegration of the deuteron near the breakup threshold.

\section{Summary}

We have tried to summarize in this report the advances in experimental techniques which have led to major recent improvements in the accuracy and reliability of electron scattering data. In our opinion the most important of these advances are those in the areas of resolution, detector systems, beam current monitoring, background suppression, the use of on-line computers, and higher counting rates (resulting largely from the development of the high-current electron 1inac). In many experiments a limiting factor at present is the difficulty of obtaining suitable (in terms of thickness, uniformity, and stability) target materials, especially in the case of separated-isotope targets. I believe that in the very near future we will see the results of the 
recent advances in experimental techniques, in the form of the greatly improved understanding of nuclear structure which can be gained by electron scattering studies. 
1. D. L. Blum, J. W. Lightbody, Jr., and S. P. Fivozinsky, experiment in progress at NBS.

2. S. Penner "Beam Handling Techniques for Electron Linear Accelerators", NBS Technical Note (to be published).

3. E. E. Chambers and R. Hofstadter, Phys. Rev. 103, 1454 (1956).

R. Belbeoch, P. Bounin, and G. Proca, J. Phys. Radium 21, 489 (1960).

4. S. Penner and J. W. Lightbody, Jr., Proc. Int. Symposium on Magnet Technology, 154, Stanford Linear Accelerator Center, Stanford, California, CONF-650922 (1965).

5. C. W. DeJager, F. Th. Duoma, P. J. T. Bruinsma, and C. DeVries, Nucl. Instr. and Methods $\underline{74}, 13$ (1969).

6. J. B. Bellicard and Ph. Leconte, Revue de Phys. Appliquee 4 , 207 (1969).

7. J. C. Bergstrom, W. Bertozzi, S. Kowalski, X. K. Maruyama, J. W. Lightbody, Jr., S. P. Fivozinsky and S. Penner, Phys. Rev. Letters 24, 4, 152 (1970).

8. S. Kowalski, W. Bertozzi, and C. P. Sargent, in Medium Energy Nuclear Physics with Electron Linear Accelerators, 39, LNS-MIT Report TID-24667 (1967).

9. S. Kowalski, private communication.

10. Harald A. Enge, Proc. Int. Symposium on Magnet Technology, 84, Stanford Linear Accelerator Center, Stanford, California, CoNF650922 (1965).

11. C. M. Braams, Nucl. Instr. and Methods 26 83 (1964).

12. J. W. Lightbody, Jr., PhD Thesis, University of Maryland, 1970 (unpublished).

13. H. W. Kenda11, Trans. on Nucl. Sci. of Inst. Radio Engrs. NS-5, 190 (1958).

14. A. Goldmann, Dissertation, Technische Hochschule, Darmstadt, 1969 (unpublished).

15. J. W. Lightbody, Jr., and S. Penner, IEEE Trans. Nucl. Sci. NS-15, 419 (1968).

16. W. R. Dodge, J. A. Coleman, S. R. Domen, and J. K. Whittaker, Rev . Sci. Instr. 37, 1151 (1966). 
17. J. Jeanjean, J. Lefrancois, J. Perez-Y-Jorba, Report L.A.L. 1143, Orsay (1965).

18. G. Charpak, R. Bouclier, T. Bressani, J. Favier, and C. Zupancic, Nuc1. Instr. and Methods $\underline{62}, 262$ (1968).

19. H. L. Crannell and L. R. Suelzle, Nucl. Instr. and Methods 44, 133 (1966).

20. R. Engfer and D. Turck, Zeit. fur Physik 205, 90 (1967).

21. D. B. Isabelle and P. H. Roy, Nuc1. Instr. and Methods 20, 17 (1963).

22. J. L. Menke, Nuc1. Instr. and Methods $\underline{64}, 61$ (1968).

23. J. L. Menke, IEEE Trans. Nucl. Sci. NS-16 921 (1969).

24. J. A. Jansen, G. J. Veenhof, and C. DeVries, Nucl. Instr. and Methods 74, 20 (1969).

25. J. F. Hague, R. E. Jennings, and R. E. Rand, Nucl. Instr. and Methods 24, 456 (1963).

26. Asher Kantz and Robert Hofstadter, Phys. Rev. $\underline{89}, 607$ (1953) and Nucleonics 12, 36 (1954).

27. Hans Dieter Wohlfahrt, Doctoral Dissertation, Johannes Gutenberg Universitat, Mainz, 1969 (unpublished).

28. Reiner Neuhausen, Doctoral Dissertation, Johannes Gutenberg Universitat, Mainz, 1969 (unpublished).

29. J. B. Broberg, IEEE Trans. on Nuc1. Sci. NS-13, 192 (1966). J. M. Wyckoff, IEEE Trans. on Nuc1. Sci. NS- $\underline{13}, 199$ (1966).

30. L. C. Maximon, Rev. Mod. Phys。 41, 193 (1969). A good bibliography of the subject of radiative corrections is included in this review article.

31. E. Romberg, N. S. Wal1, D. Blum, J. Lightbody, Jr., and S. Penner, Bul1. Am. Phys. Soc. 14, 12, abstract FC14, (Dec. 1969).

32. D. G. Ravenha11, private communication.

33. J. C. Bergstrom, in Medium Energy Nuclear Physics with Electron Linear Accelerators, 251, LNS-MIT Report TID-24667 (1967).

34. L. C. Maximon and D. B. Isabelle, Phys. Rev. 133, B1344 (1964). L. C. Maximon and D. B. Isabelle, Phys. Rev. 136, B674 (1964). Y. S. Tsai, Int. Conf. on Nucleon Structure, Stanford Univ. Press, Stanford, California (1965). 
35. S. T. Tuan, L. E. Wright, and D. S. Onley, Nuc1. Instr and Methods 60, 70 (1968).

C. R. Fischer and G. H. Rawitscher, Phys. Rev. 135, B377 (1964).

D. Drechse1, Nuc1. Phys. A113, 665 (1968).

T. H. Schucan, Phys. Rev. 171, 1142 (1968).

36. R. E. Rand, R. Frosch, and M. R. Yearian, Phys. Rev. Letters 14, 234 (1965).

J. Goldemberg, L. A. L. 1140, Orsay (1965).

37. R. A. Schrack, Nuc1. Instr. and Methods $\underline{45}, 319$ (1966).

38. H. Cranne11, C. J. Cranne11, F. J. K1 ine, J. T. O'Brien, S. P. Fivozinsky, and S. Penner, Bul1. Am. Phys. Soc. 14, 12, abstract FC 10 (Dec. 1969).

39. Note added in proof: Very recent work at Saclay indicates that the count rate limitation of multiwire proportional chambers can be improved by the use of electronegative gases in the chambers. This group has observed resolution times near 30ns and counting rates of the order of $10^{7}$ per second. See: "Multiwire Proportiona1 and Semiproportional Counter with a Variable Sensitive Volume" C. Grunberg, L. Cohen, and L. Mathieu, preprint, Service de Physique Nucleaire a Haute Energie, Centre d'E tudes Nucleaires de Saclay. We thank Prof. W. Bertozzi for calling this paper to our attention. 
Table I

\section{EFFECTS CONTRIBUTING TO OBSERVED RESOLUTION}

The resolution factors 1 isted are deduced from experimental data obtained with the NBS electron scattering facility. The percentages are given as full width at half maximum (FWHM) values relative to the incident beam energy. The effects 1 isted should be added in quadrature to obtain the overa11 resolution (.07 percent, minimum). The starred entries are the FWHM resolutions of gaussian distributions which would give the same contributions as the actual rectangular distributions $\frac{37 /}{}$. The target referred to here is $20 \mathrm{mg} / \mathrm{cm}^{2}$ polyethylene, $\left(\mathrm{CH}_{2}\right)_{\mathrm{n}}$, and the values quoted are for incident energies 60-100 MeV and scattering angles 90-145 degrees.

FACTOR

I. Beam Transport

Entrance Aperture

Energy Slits

Intrinsic

II. Spectrometer

Detector Size

Intrinsic

Beam Spot Size

III. Target

Kinematics

Energy Loss Straggling
PERCENT

$$
\begin{array}{r}
.04-.09 * \\
\text { varies } \\
.04
\end{array}
$$

$.03 *$

.03

.02

$.01-.02 *$

$.01-.02$ 

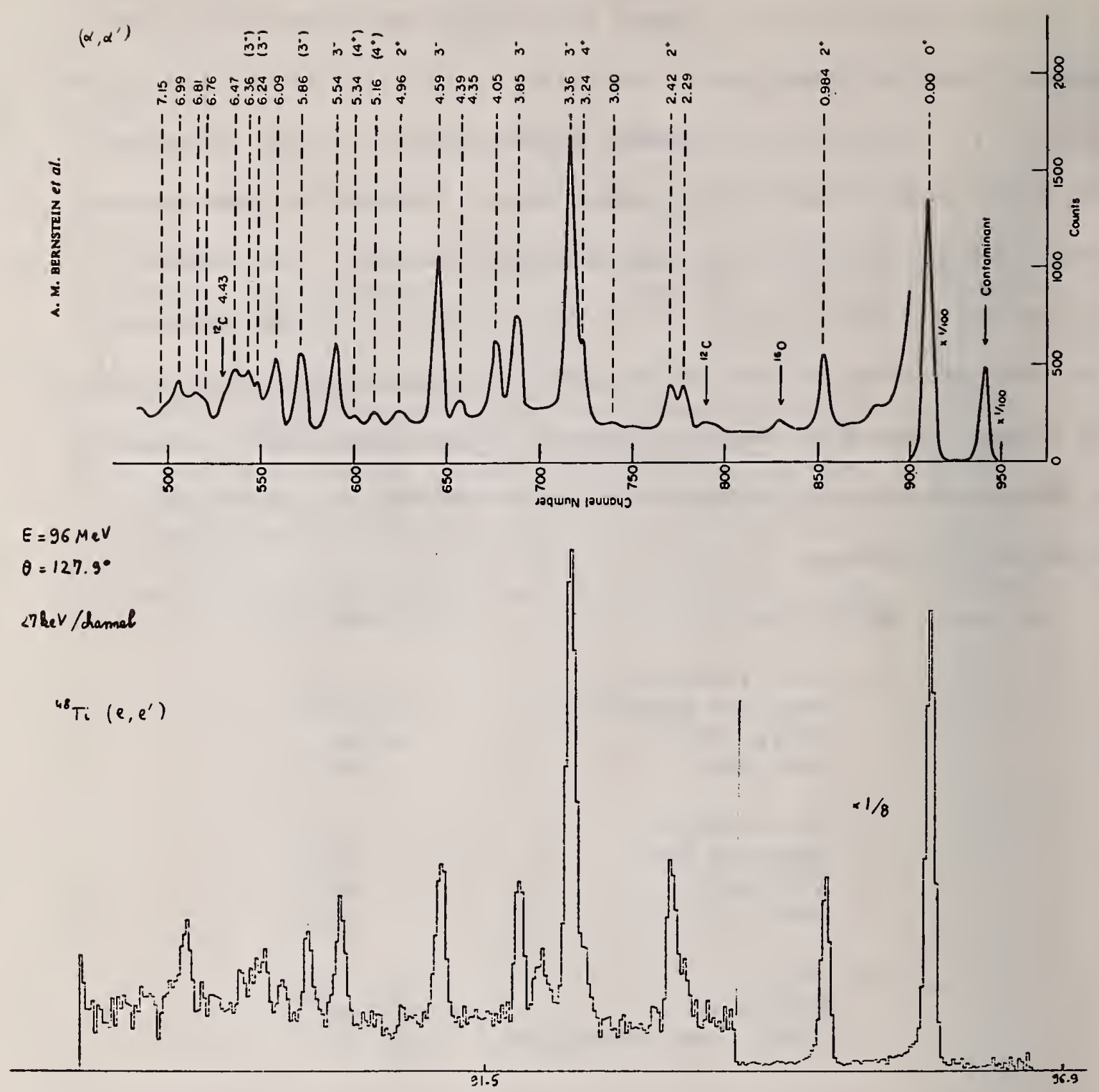

Figure 1. Spectrum of electrons scattered from $\mathrm{Ti}^{48}$ (lower figure) compared to alpha particle spectrum from the same nucleus. The energy scales for the two figures have been made equal. Vertical scales are arbitrary. The $\left(\alpha, \alpha^{\prime}\right)$ data is taken from the paper of A. M. Bernstein, et al., Nuc1. Phys. A115, 79 (1968). 


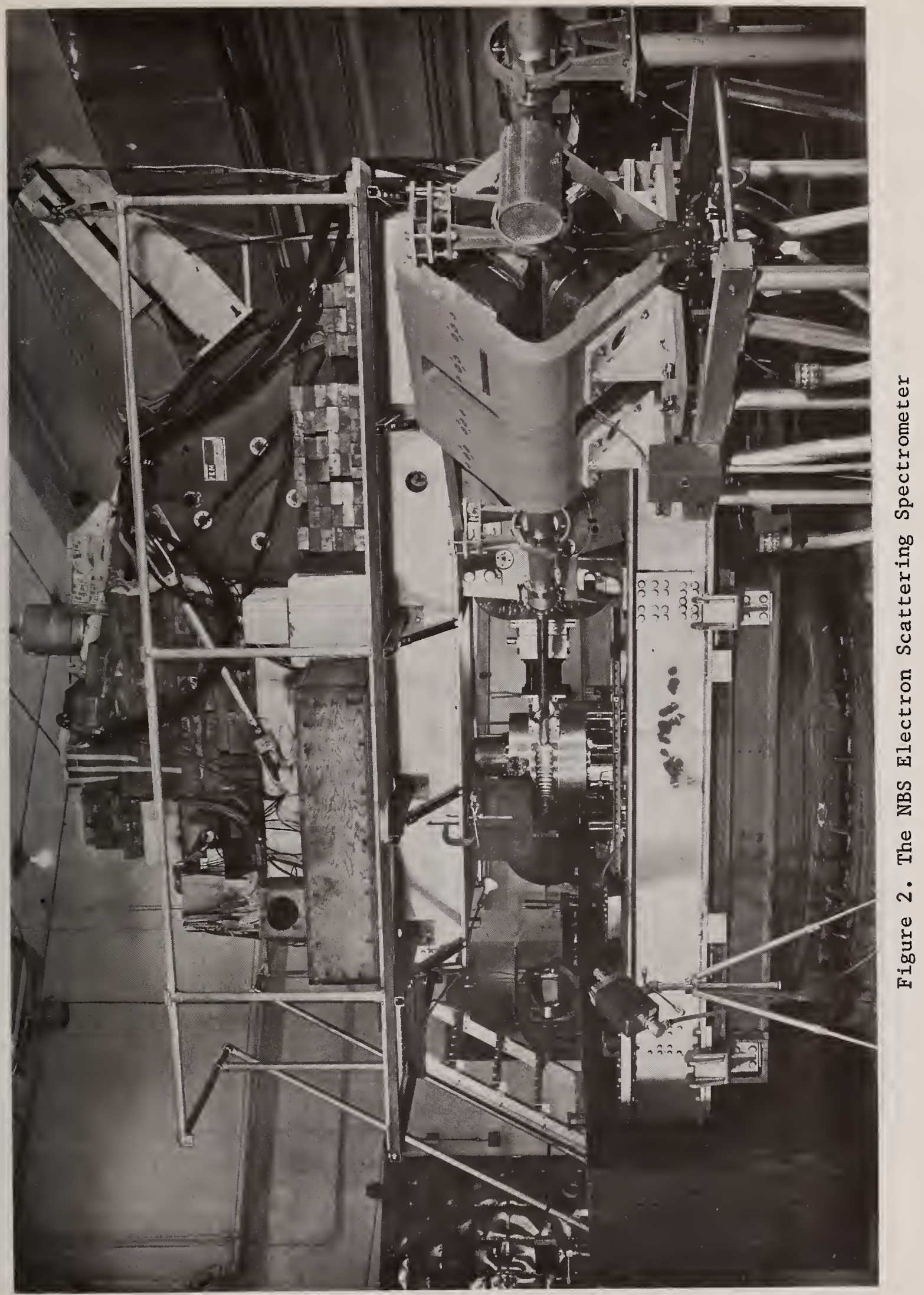




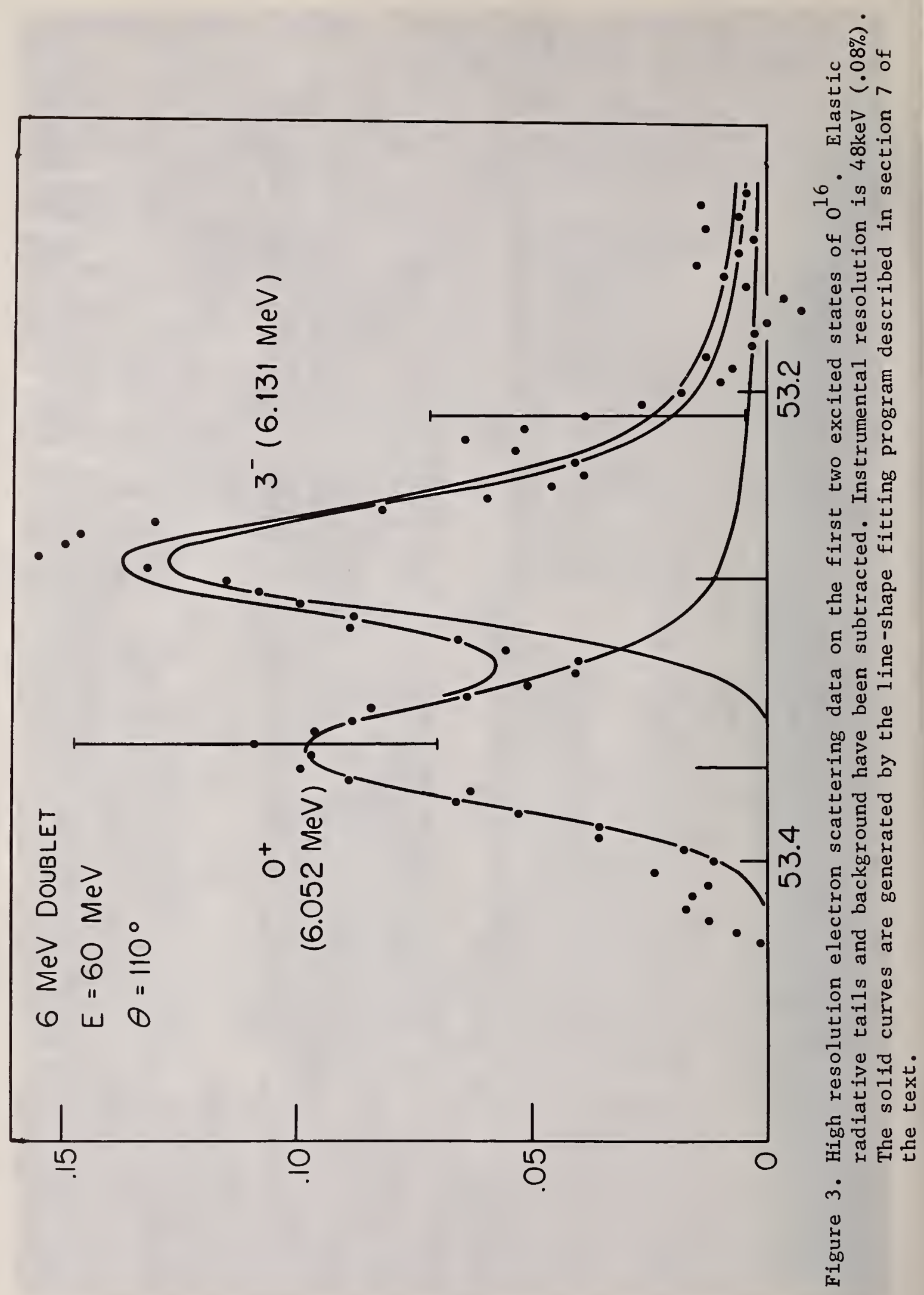




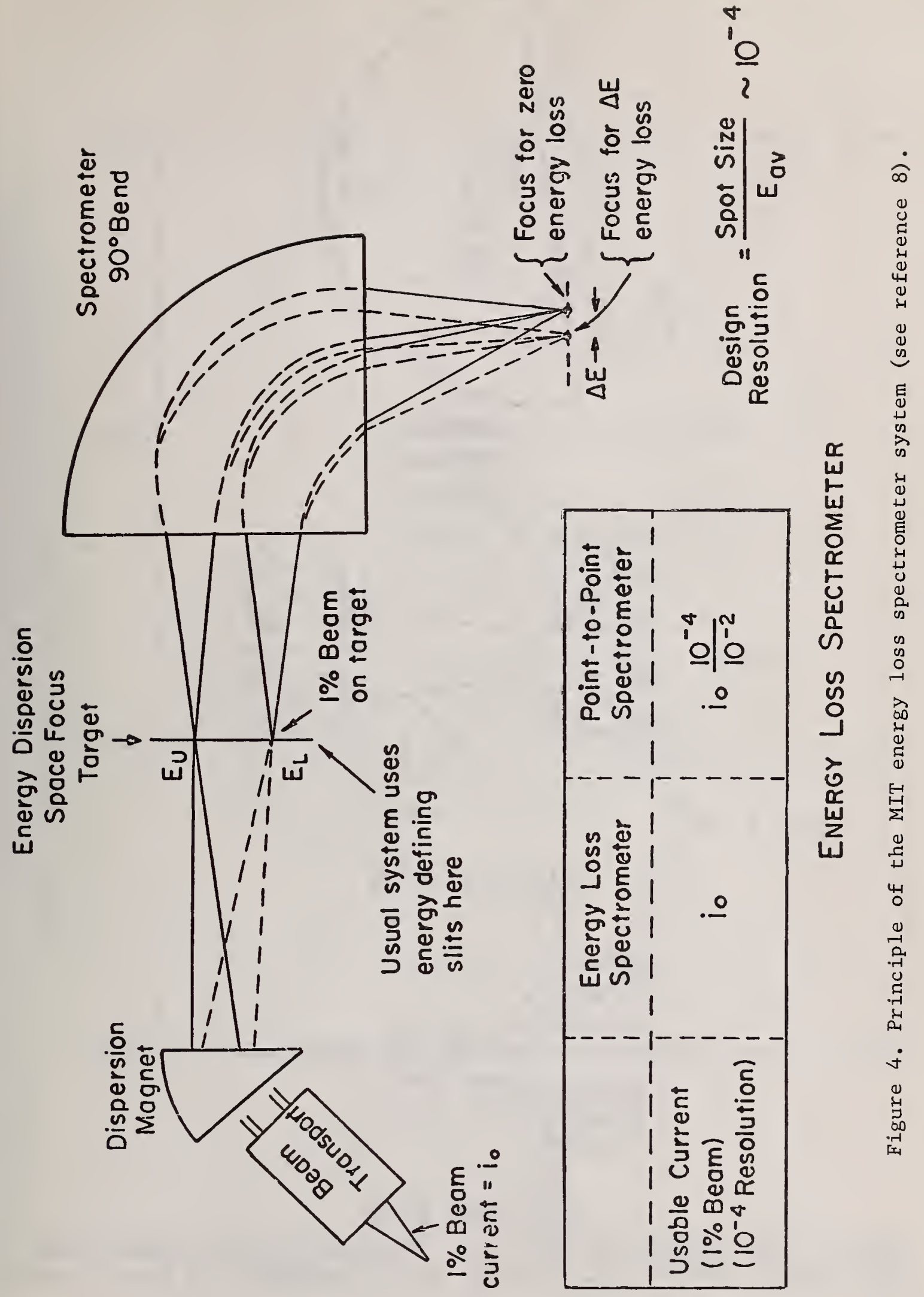




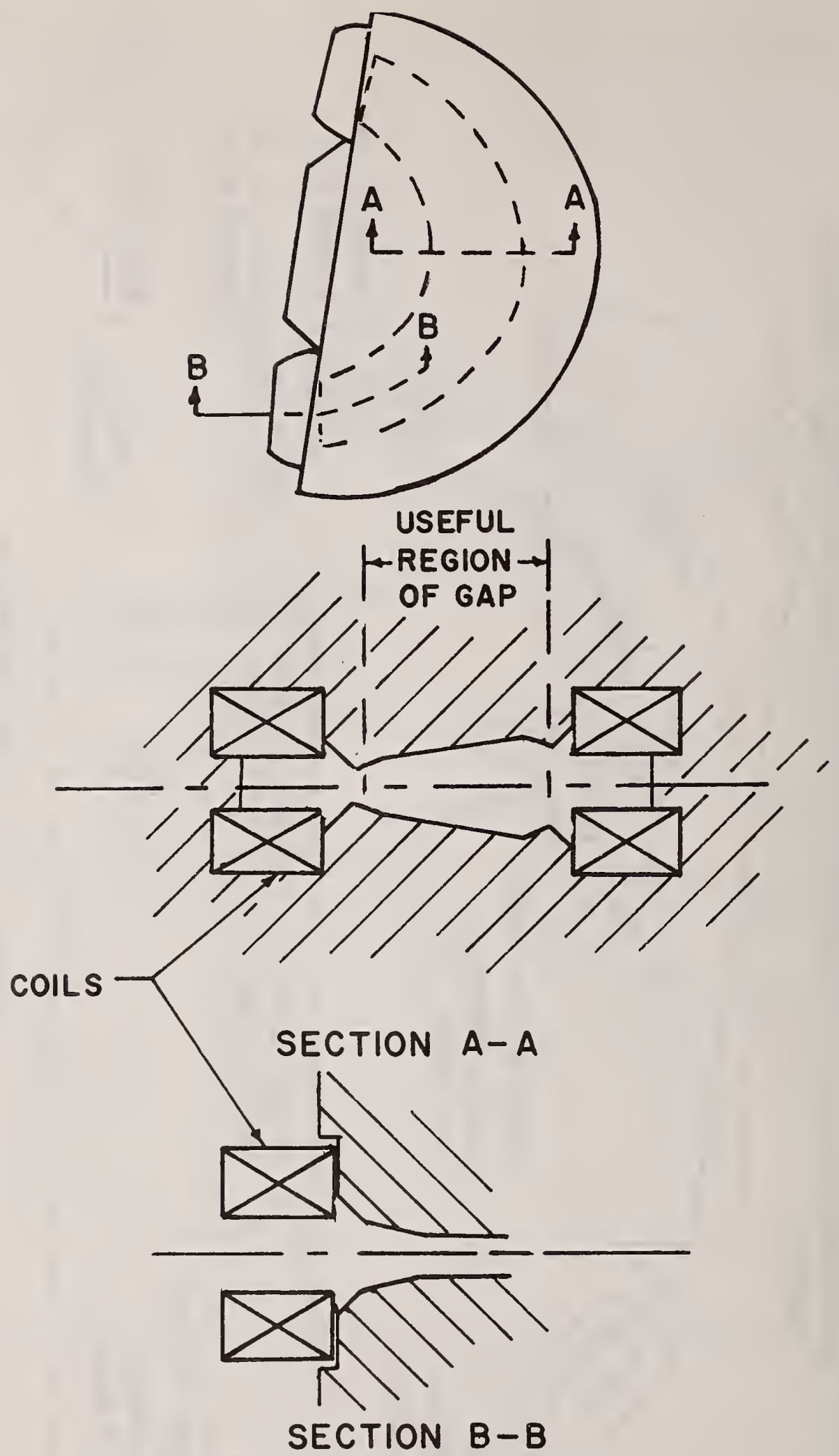

Figure 5. NBS spectrometer pole shape, illustrating the chamfered edges which result in a field distribution nearly independent of field strength. 


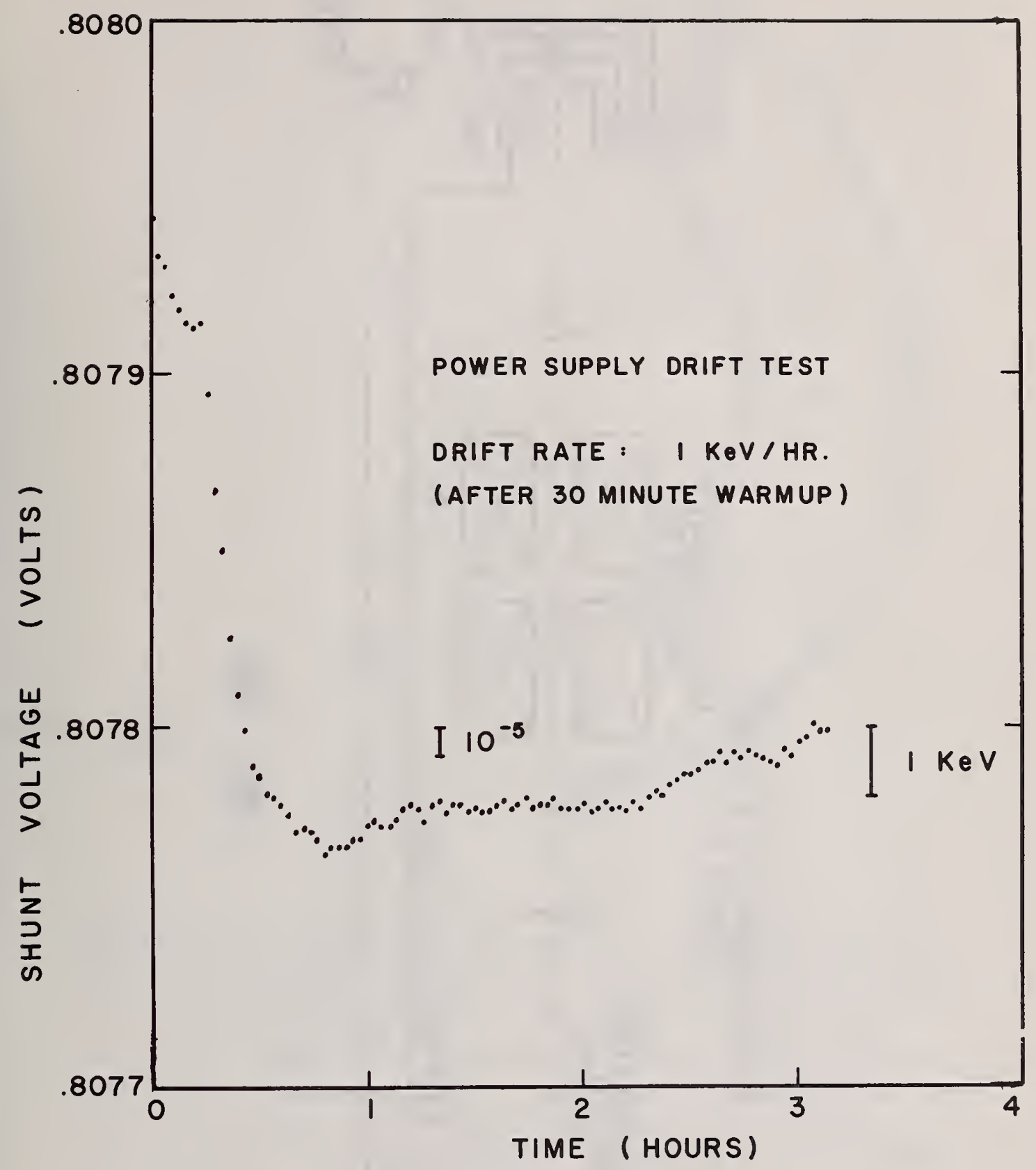

Figure 6. Current stability test of the NBS spectrometer and power supply. The labelled vertical bars show the shunt voltage changes which correspond to one part in $10^{5}$ and one $\mathrm{keV} / \mathrm{C}$ momentum changes. 


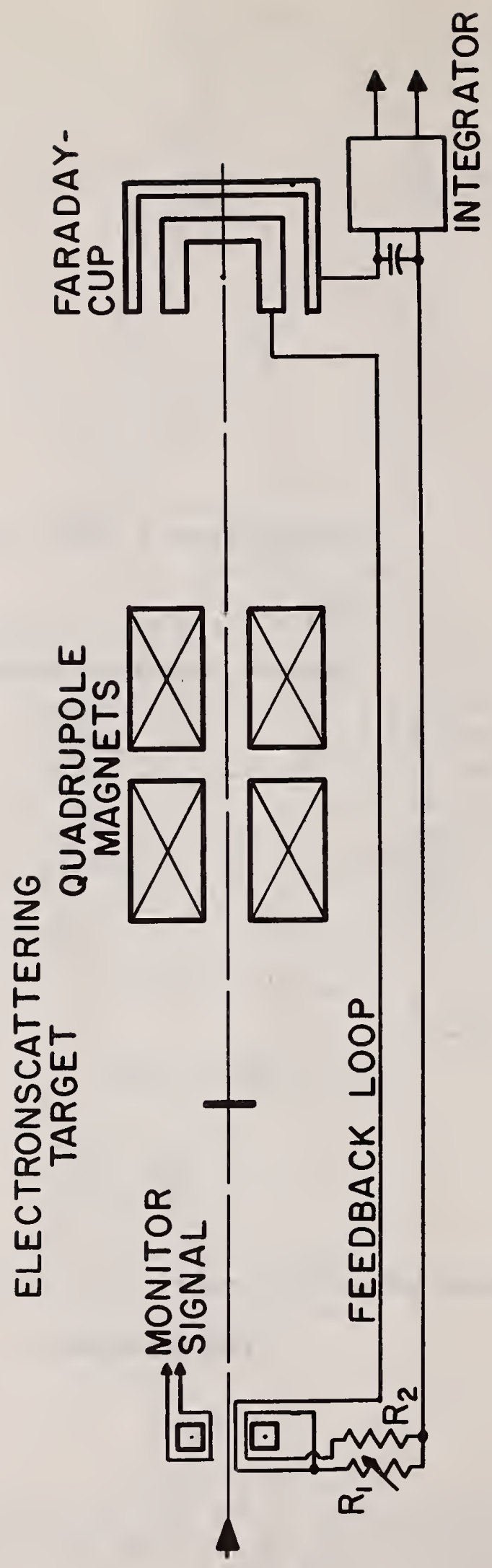

ป

离

\&

ثี

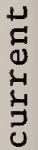

ฐ్

్ㅠㅁ

崩

荌

E्]

N

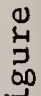

II 


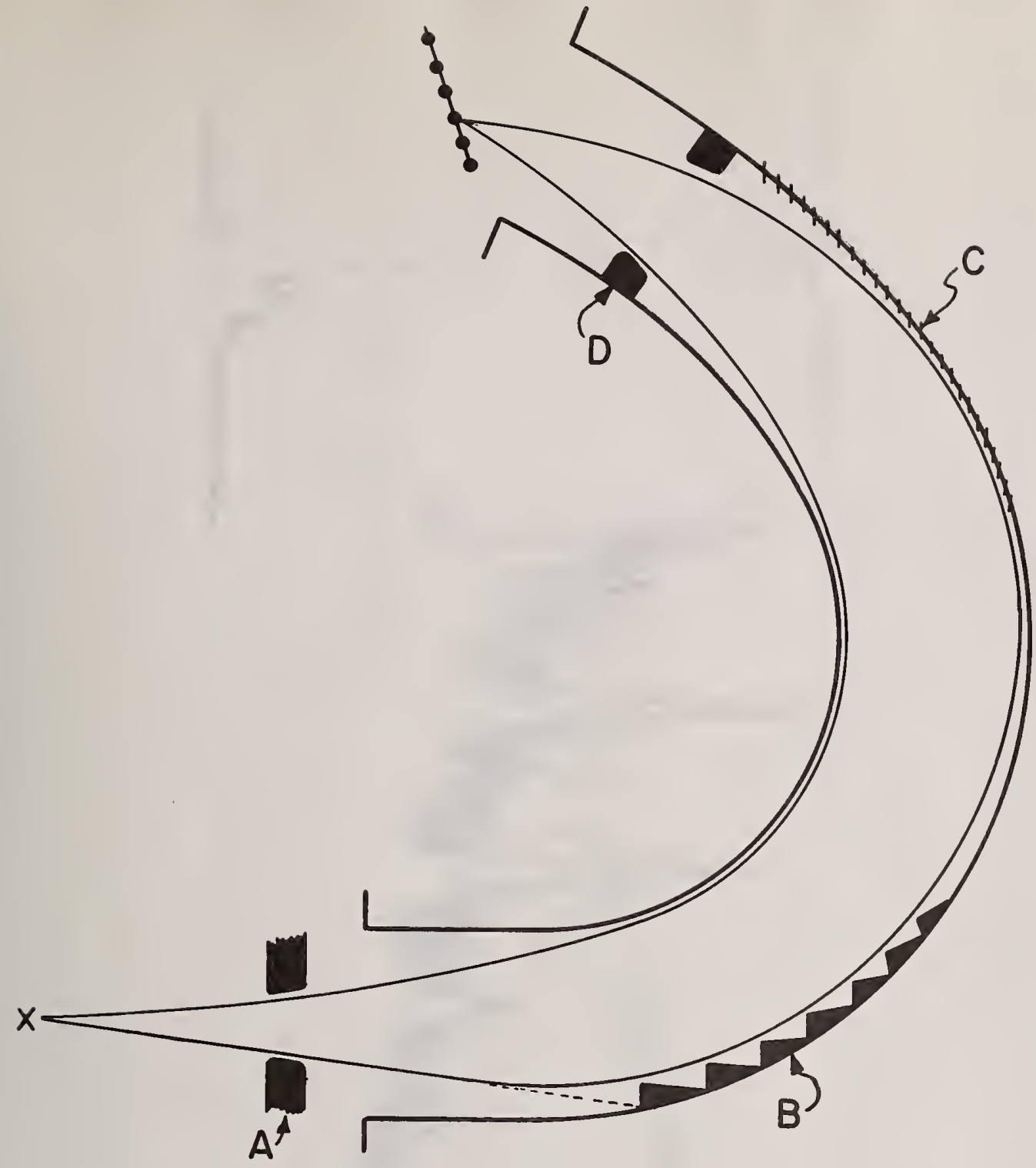

Figure 8. Baffling system on the NBS spectrometer. A tungsten and lead collimator at A defines the vertical acceptance angle and reduces the horizontal acceptance at this point. (The definition of horizontal acceptance is provided by the vacuum chamber wa11s.) The lead "steps" at B are intended to absorb high energy (e.g. elastically scattered) electrons when the magnetic field of the spectrometer is set for transmission of low energy electrons. A low $-\mathrm{Z}$ liner at $\mathrm{C}$ reduces scattering of electrons from the vacuum chamber. The lead aperture at D prevents electrons from striking the vacuum chamber near the detectors and also absorbs electrons previously scattered from the vacuum chamber. Aperture $D$ does not restrict the solid angle for electrons headed towards the detectors. These measures together reduce the instrumental background by more than a factor of ten. 


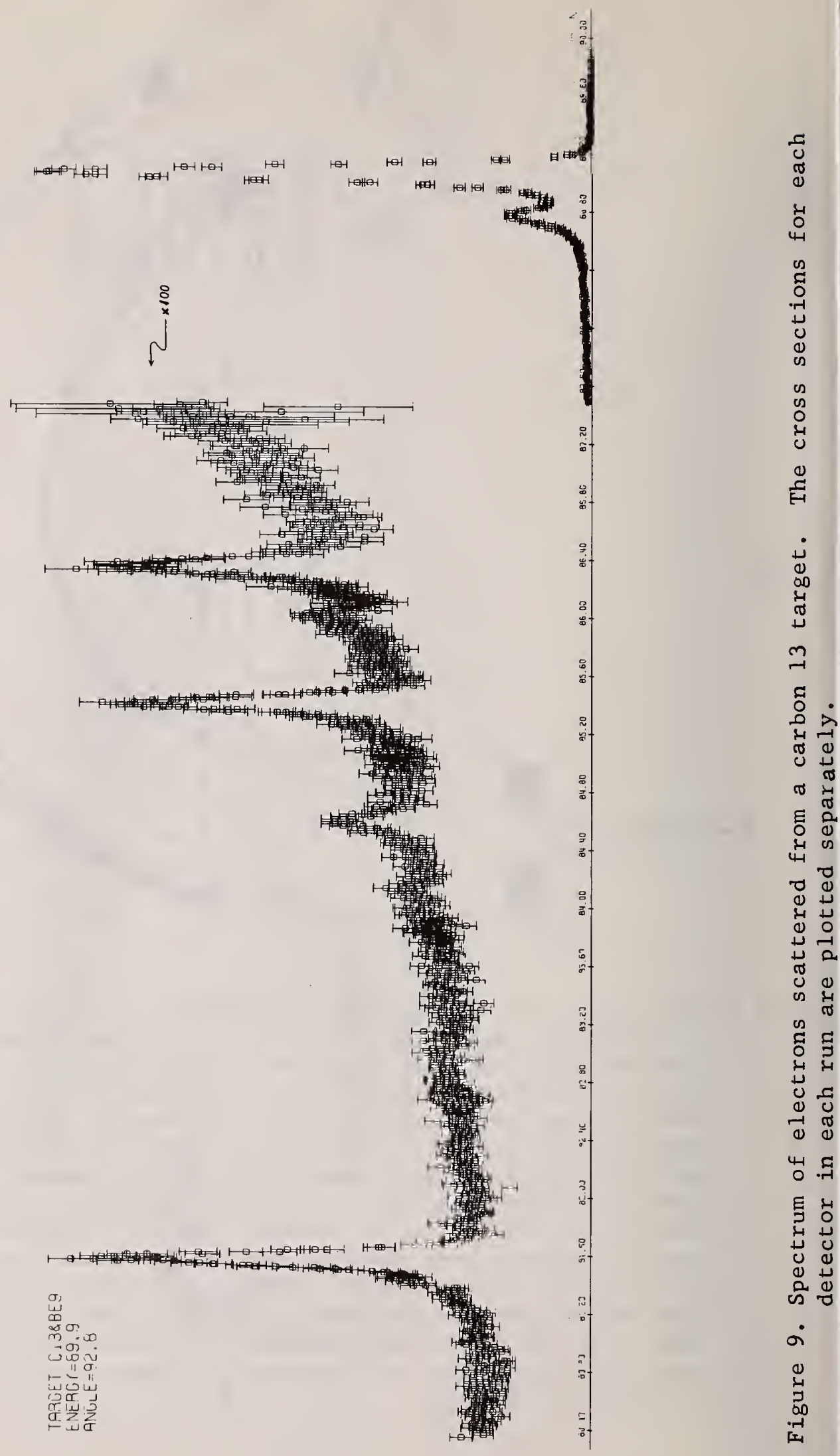




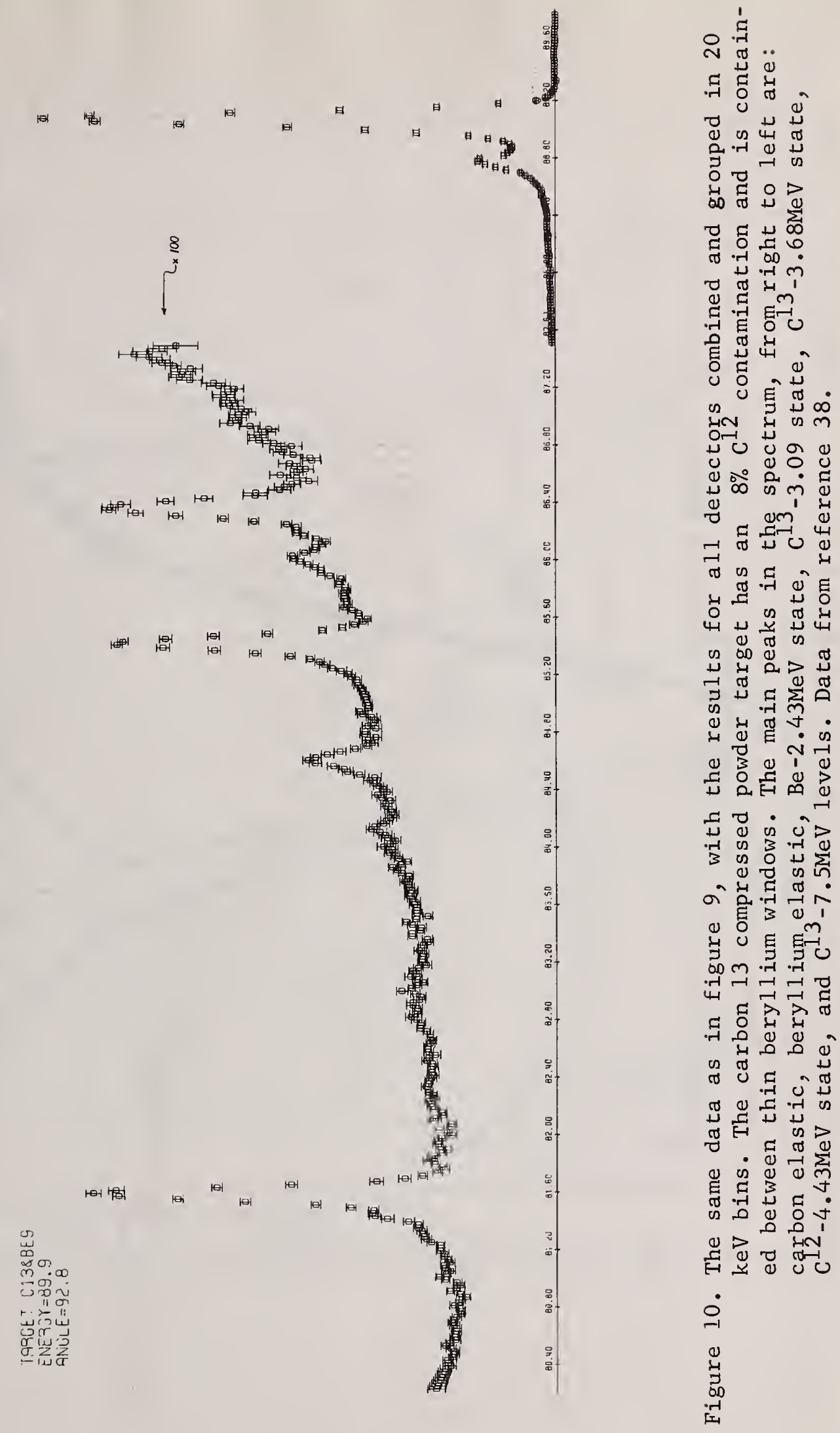




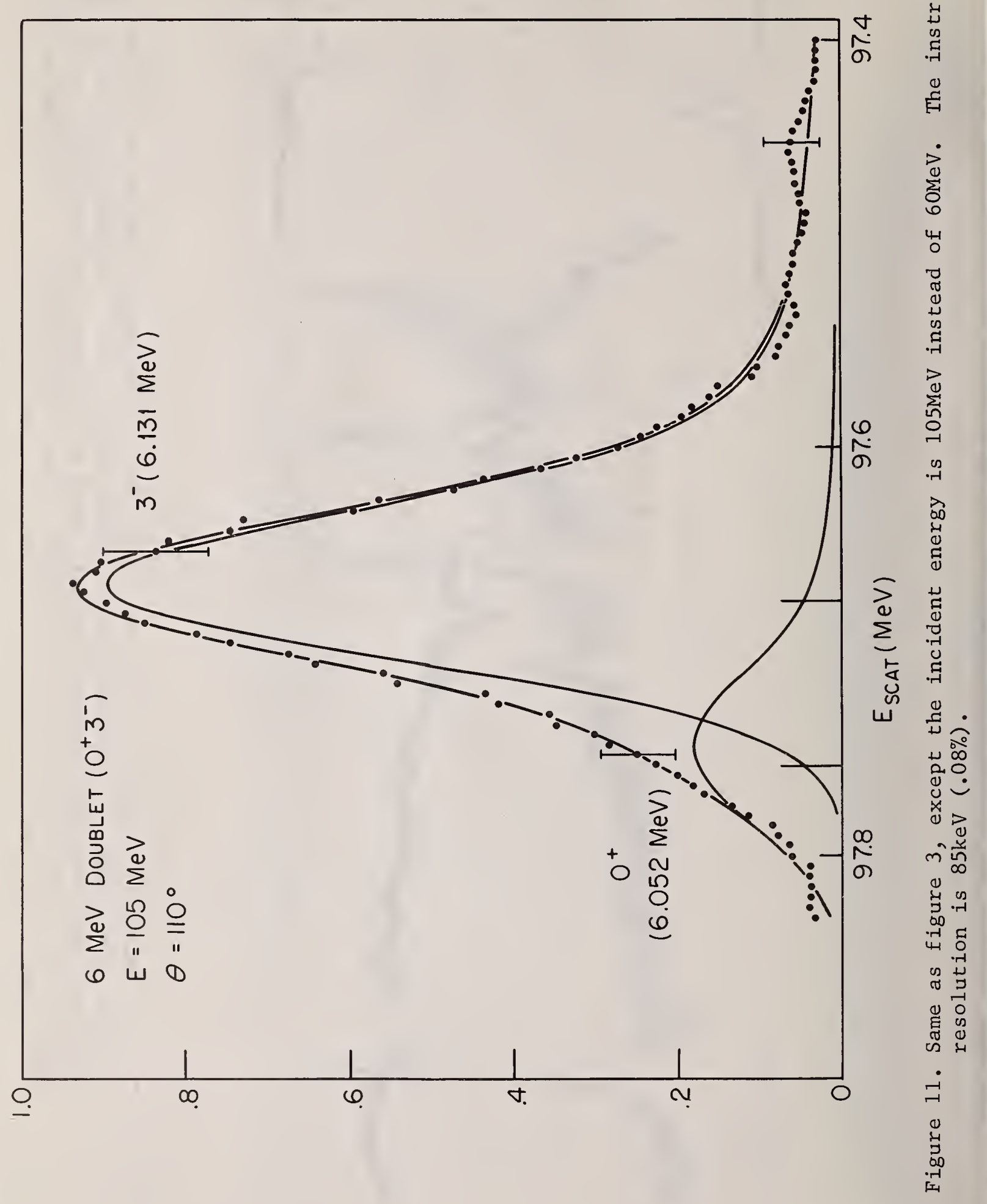




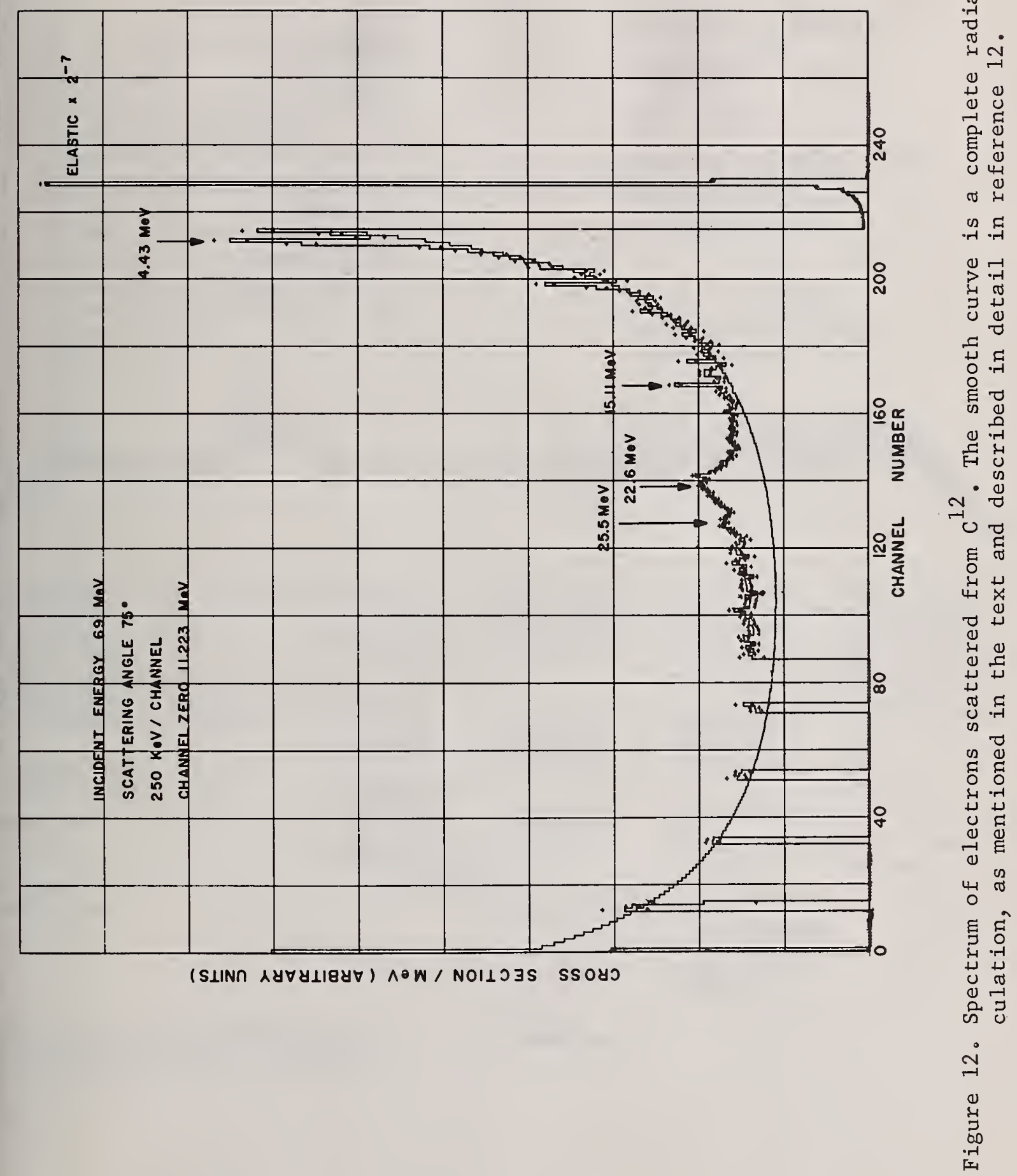



THE NATIONAL ECONOMIC GOAL

Sustained maximum growth in a free market economy, without inflation, under conditions of full employment and equal opportunity

\section{THE DEPARTMENT OF COMMERCE}

The historic mission of the Department is "to foster, promote and develop the foreign and domestic commerce" of the United States. This has evolved, as a result of legislative and administrative additions, to encompass broadly the responsibility to foster, serve and promote the nation's economic development and technological advancement. The Department seeks to fulfill this mission through these activities:

Participating with other government agencies in the creation of national policy, through the President's Cabinet and its subdivisions.

- Cabinet Committee on Economic Policy
Promoting progressive Assisting states, business policies and communities and growth.

- Business and Defense Services Administration

- Office of Field Services
- Urban Affairs

Council

- Environmental

Quality Council

NOTE: This schematic is neither an organization chart nor a program outline for budget purposes. It is a general statement of the Department's mission in relation to the national goal of economic development.

JuLY 1969
Strengthening the international economic position of the United

States.

- Bureau of International Commerce

- Regional Planning Commissions

- Office of Minority Business Enterpriso

- Office of Foreign Commercial Services

- Office of Foreign Direct Investments

- United States Travel Service

- Maritime Administration

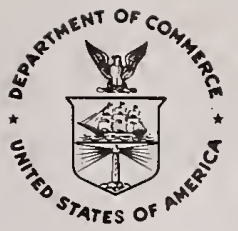

MISSION AND FUNCTIONS OF THE DEPARTMENT OF COMMERCE
"to foster, serve and promote the nation's economic development and technological advancement"

Assuring effective Acquiring, analyzing use and growth of the and disseminating nation's scientific information concernand technical ing the nation and resources. the economy to help achieve increased

- Environmental social and economic Science Services benefit. Administration

- Patent Office

- Bureau of the Census

- National Bureau of - Office of Business Standards Economics

- Office of Telecommunications

- Office of State Technical Services 



\section{PERIODICALS}

JOURNAL OF RESEARCH reports National Bureau of Standards research and development in physics, mathematics, chemistry, and engineering. Comprehensive scientific papers give complete details of the work, including laboratory data, experimental procedures, and theoretical and mathematical analyses. Illustrated with photographs, drawings, and charts

Published in three sections, available separately:

\section{- Physics and Chemistry}

Papers of interest primarily to scientists working in these fields. This section covers a broad range of physical and chemical research, with major emphasis on standards of physical measurement, fundamental constants, and properties of matter. Issued six times a year. Annual subscription: Domestic, $\$ 9.50$; for eign, $\$ 11.75^{*}$.

\section{Mathematical Sciences}

Studies and compilations designed mainly for the mathematician and theoretical physicist. Topics in mathematical statistics, theory of experiment design, numerical analysis, theoretical physics and chemistry, logical design and programming of computers and computer systems. Short numerical tables. Issued quarterly. Annual subscription: Domestic, $\$ 5.00$; foreign, $\$ 6.25^{*}$.

\section{Engineering and Instrumentation}

Reporting results of interest chiefly to the engineer and the applied scientist. This section includes many of the new developments in instrumentation resulting from the Bureau's work in physical measurement, data processing, and development of test methods. It will also cover some of the work in acoustics, applied mechanics, building research, and cryogenic engineering. Issued quarterly. Annual subscription: Domestic, $\$ 5.00$; foreign, $\$ 6.25 *$.

\section{TECHNICAL NEWS BULLETIN}

The best single source of information concerning the Bureau's research, developmental, cooperative and publication activities, this monthly publication is designed for the industry-oriented individual whose daily work involves intimate contact with science and technology-for engineers, chemists, physicists, research managers, product-development managers, and company executives. Annual subscription: Domestic, $\$ 3.00$; foreign, $\$ 4.00^{*}$.

\section{NONPERIODICALS}

Applied Mathematics Series. Mathernatical tables, manuals, and studies.

Building Science Series. Research results, test methods, and performance criteria of building materials, components, systems, and structures.

Handbooks. Recommended codes of engineering and industrial practice (including safety codes) developed in cooperation with interested industries, professional organizations, and regulatory bodies.

Special Publications. Proceedings of NBS conferences, bibliographies, annual reports, wall charts, pamphlets, etc.

Mornographs. Major contributions to the technical literature on various subjects related to the Bureau's scientific and technical activities.

National. Standard Reference Data Series. NSRDS provides quantitive data on the physical and chemical properties of materials, compiled from the world's literature and critically evaluated.

Product Standards. Provide requirements for sizes, types, quality and methods for testing various industrial products. These standards are developed cooperatively with interested Government and industry groups and provide the basis for common understanding of product characteristics for both buyers and sellers. Their use is voluntary.

Technical Notes. This series consists of communications and reports (covering both other agency and NBS-sponsored work) of limited or transitory interest.

Federal Information Processing Standards Publications. This series is the official publication within the Federal Government for information on standards adopted and promulgated under the Public Law 89-306, and Bureau of the Budget Circular A-86 entitled, Standardization of Data Elements and Codes in Data Systems.

\section{CLEARINGHOUSE}

The Clearinghouse for Federal Scientific and Technical Information, operated by NBS, supplies unclassified information related to Government-generated science and technology in defense, space, atomic energy, and other national programs. For further information on Clearinghouse services, write:

Clearinghouse

U.S. Department of Commerce Springfield, Virginia 22151

- Difference in price is due to extra cost of foreign mailing.

Order NBS publications from:

Superintendent of Documents

Government Printing Office

Washington, D.C. 20402 
U.S. DEPARTMENT OF COMMERCE

WASHINGTON, D.C. 20230

OFFICIAL BUSINESS

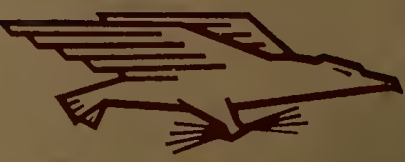

